\title{
Persistent current generation by the spin-vortex formation in the cuprate with the single-valuedness constraint on the conduction electron wave functions
}

Division of Materials Science, University of Tsukuba,Tsukuba, Ibaraki 305-8573, Japan

\author{
Hiroyasu Koizumi, Ryo Hidekata, Akira Okazaki \\ Department of Physics, Tohoku University, Sendai, Miyagi 980-8578, Japan
}

\section{Masashi Tachiki}

\begin{abstract}
The persistent current generation in the cuprate is theoretically investigated based on the spin-vortex superconductivity theory. We present a way to impose the single-valued condition on wave functions and clarify the appearance of a vector potential and the persistent current generation by it when the spin-vortices are created by the conduction electrons. We attribute the reason for the very high superconducting transition temperature in the cuprate to the enhanced stability of the spin-vortices by the strong hole-lattice interaction.
\end{abstract}

\section{Introduction}

It is now widely believed that the dual roles played by the conduction electrons, namely charge carriers and spin-degree-of-freedom holders, lead the cuprate to a superconductor $[1,2,3,4,5,6,7,8,9,10,11,12,13,14]$. In this respect, it is noteworthy that a number of works have shown that spin-vortices are created by the conduction electrons in the antiferromagnetic background in the cuprate $[7,8,9,10,11,12,13,14]$. A novel superconductivity theory, spin-vortex superconductivity, is also proposed as a theory that explains the superconductivity in the cuprate based on the spin-vortex formation[15, 16].

The spin-vortices are topological defects in the spin configuration. A number of theoretical investigations have considered the spin-vortex formation with spins lying in the $\mathrm{CuO}_{2}$ plane $[9,10,11,12,13,14,15,16]$. When the conduction electrons make the Hartree-Fock field with the spin-vortices lying in the $\mathrm{CuO}_{2}$ palne, the spin direction of the self-consistent field is described by angular variable $\xi$ with period $2 \pi$. This variable $\xi$ also describes the twisting of the spin of the conduction electron moving in the HartreeFock field. The fluctuation of the spins that are not included in the Hartree-Fock field will smear the spin vortices in the spin configuration; however, the topological characters of the spin-vortices will remain intact since they cannot be altered in an arbitrary manner. In the present work, we concern the topological characters of the 
spin-vortices and the twisting of the spin basis of the conduction electron wave function caused by them.

It has been anticipated that the doped holes are the carriers of the persistent current in the cuprate. However, at low temperatures, the mobility of the holes in the bulk is significantly reduced by the small polaron formation by the strong hole-lattice interaction $[17,18,19]$. The small polaron formation of the hole with changing the $\mathrm{O}-\mathrm{Cu}$ bond length has been detected by the XAFS experiments [20]; the observed value of the $\mathrm{O}-\mathrm{Cu}$ bond length change agrees with the ab initio molecular orbital cluster calculation [21]. According to the latter, the stabilization of the hole with the lattice deformation makes the hole essentially immobile. This indicates that doped holes cannot be the charge carriers of the persistent current; in this respect, it is noteworthy that the Hall coefficient measured below the superconducting transition temperature $T_{c}$ by destroying the superconducting state with a strong magnetic field is negative, although it is positive at the room temperature [22]; this result may be interpreted that the mobility of the holes is much reduced at low temperatures due to the small polaron formation. Note that the small polaron formation is probably suppressed in the surface region since such effects are minor in the ARPES measurements [23]; the surface electronic state is probably somewhat different from the bulk electronic state.

The purpose of the present work is to clarify the recently proposed novel persistent current generation mechanism that occurs when the doped holes form immobile small polarons and become the cores of the spin-vortices. This mechanism attributes the ultimate cause of the persistent current generation to the single-valuedness of wave functions; ever since Schrödinger solved the Schrödinger equation for the hydrogen atom [24], the single-valuedness of wave functions is one of the fundamental postulates of quantum mechanics; he required this condition and obtained the correct discrete values of the energy levels. One might think that the single-valued condition on wave functions is trivially satisfied; however, it is not so when the wave functions are multi-component. The conduction electrons have the spin-degree-of-freedom, thus, their wave functions are two component. When the twisting of the spin basis of the conduction wave function occurs, the sign-change is brought about by the twisting. This sign-change makes the construction of the single-valued wave functions nontrivial.

Actually, a similar problem is encountered in the study of molecular systems using the Born-Oppenheimer approximation. In this approximation, two-component electronic wave functions appear around the conical intersection of the potential energy surface. The conical intersection is a singularity of the Born-Oppenheimer approximation, and the sign-change of electronic wave functions occurs when they are transported around it $[25,26]$. This sign change makes the construction of single-valued total wave functions nontrivial. Mead showed that this problem can be handled by introducing a $U(1)$ phase factor that compensates the sign-change [27]; the added phase factor induces a vector potential, and the induced vector potential brings about an Aharonov-Bohm type effect [28], called the 'molecular Aharonov-Bohm effect'.

We will put forward a method that handles the above mentioned sign-change, 


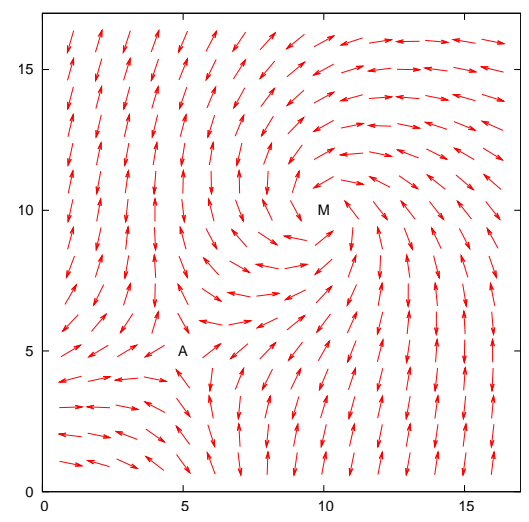

Figure 1. Two spin-vortices in the antiferromagnetic background. Spin-vortices in the $16 \times 16$ lattice. ' $M$ 'and ' $A$ ' indicate cores of spin-vortices with winding numbers +1 and -1 in Eq. (4), respectively. We may call the one with the winding number -1 the anti-vortex.

properly; namely, we present a way to impose the single-valuedness constraint on the conduction electron wave function with the twisted spin basis. From the single-valued constraint, the persistent current is generated.

Let us describe the organization of the present work. In Section 2, we clarify the persistent current generation that arises from the single-valued constraint on the wave functions. We impose the constraint by introducing a $U(1)$ phase factor $e^{-i \chi / 2}$, where $\chi$ is a harmonic function of period $2 \pi$; the Lagrange multipliers are used to force the phase $\chi$ to compensate the sign-change caused by the spin twisting. Consequently, the variational construction of the wave functions are done by the energy minimization with the constraint.

In Section 3, the energy functional that depends on the gradient of the $U(1)$ phase factor introduced above, i.e., $\nabla \chi$, is constructed. Using this energy functional, the Lagrange multipliers and the optimized $\nabla \chi$ are obtained from the stationary condition of the functional that is the sum of the energy functional and the constraint. Using the optimized $\nabla \chi$, the single-valued wave functions are constructed.

In Section 4, an example calculation is worked out. In Section 5, the relation between the present theory and the London's theory of superconductivity is examined. Lastly, in Section 6, we will discuss the stabilization of the spin-vortices by the strong hole-lattice interaction in the hole-doped cuprate, and conclude the present work.

\section{Persistent current generation by the single-valued wave function constraint}

At low temperatures where the mobility of the holes is very small, the number of the accessible sites and that of the conduction electrons are equal. The classical spin 
Hamiltonian for this situation is given by

$$
E[\xi]=\frac{J}{4} \sum_{\langle i, j\rangle_{1} ; i, j \in \text { acc. sites }} \cos \left(\xi_{i}-\xi_{j}\right)
$$

where 'acc. sites' in the sum indicates a set of sites 'accessible' for electrons, and the hole occupied sites are simply omitted as the inaccessible sites by assuming that the polaron hopping is negligible; $\xi_{j}$ is the value of the angle $\xi$ at the $j$ th site, $J$ is the super-exchange coupling constant between the spins, $\langle i, j\rangle_{1}$ indicates the nearest neighbor pairs, and the spins are assumed to lie in the $\mathrm{CuO}_{2}$ plane (the $x$-y plane) as is observed experimentally in the parent compound [29].

The energy function in Eq. (1) has a lot of local minima that differ in the number, positions, and winding numbers of spin-vortices. The spin-vortices are created with the holes at their centers since in this way the core energy of the spin-vortex is reduced [12]. An example for the local minimum spin configuration numerically calculated using Eq. (1) is depicted in Fig. 1, in which two spin-vortices are embedded in the antiferromagnetic background with holes at their centers.

The energy function in Eq. (1) is very similar to the 2D XY model, in which vortex-antivortex pairs are frozen-in below the transition temperature $T_{\mathrm{BKT}}[30,31]$. We consider the situation where the frozen-in spin-vortex and antivortex pairs exist with the holes at their centers.

When the conduction electrons move in the self-consistent field with the frozen-in spin-vortices, the conduction electron wave functions contain the following phase factors,

$$
e^{ \pm i \frac{\xi_{j}}{2}}
$$

The angular variable $\xi$ may have the jump of value $\xi_{j} \rightarrow \xi_{j}+2 \pi n$, where $n$ is an integer; then, the phase factor in Eq. (2) may make the wave functions multi-valued. For example, if the jump of the $\xi_{j}$ value is $2 \pi$ times an odd number, the sign-change occurs,

$$
e^{ \pm i \frac{\xi_{j}}{2}} \rightarrow-e^{ \pm i \frac{\xi_{j}}{2}}
$$

Spin-vortices lying in the $x-y$ plane are characterized by the topological index, the winding number, defined by

$$
w[\xi]_{\ell}=\frac{1}{2 \pi} \oint_{C_{\ell}} \nabla \xi \cdot d \mathbf{r},
$$

where $C_{\ell}$ is a loop in the $x$ - $y$ plane.

Actually, since we are considering the discrete lattice system, the above integration becomes the sum

$$
w[\xi]_{\ell}=\frac{1}{2 \pi} \sum_{i=1}^{N_{\ell}}\left(\xi_{C_{\ell}(i+1)}-\xi_{C_{\ell}(i)}\right),
$$

where $N_{\ell}$ is the total number of sites on the loop $C_{\ell}$, and $C_{\ell}(i)$ is the $i$ th site on it with the periodic condition $C_{\ell}\left(N_{\ell}+1\right)=C_{\ell}(1)$.

If some of $w[\xi]]_{\ell}$ 's are odd, the phase factors in Eq. (2) may make the wave functions multi-valued since the transportation of $\xi$ along the loop $C_{\ell}$ with the odd winding 
number $w[\xi]_{\ell}$ will yield a phase shift of $2 \pi$ times an odd number; in this case the sign change of $e^{ \pm i \frac{\xi_{j}}{2}}$ given in Eq. (3) occurs after the transportation.

This sign-change makes the construction of the single-valued wave functions nontrivial. Mead showed that this sign-change can be handled by introducing a $U(1)$ phase factor [27]. We follow Mead and use a $U(1)$ phase factor to obtain the singlevalued wave functions; i.e., we introduce an angular variable $\chi$ with period $2 \pi$, and modify the phases in Eq. (2) as

$$
e^{ \pm i \frac{\xi_{j}}{2}} e^{-i \frac{\chi_{j}}{2}}
$$

The added phase factor is considered as a variational parameter that has to be optimized.

Actually, as will be shown later, the conduction electron wave function is obtained in the following form

$$
\sum_{j} e^{-i \frac{\chi_{j}}{2}}\left(e^{-i \frac{\xi_{j}}{2}} C_{j \uparrow} c_{j \uparrow}^{\dagger}+e^{i \frac{\xi_{j}}{2}} C_{j \uparrow} c_{j \downarrow}^{\dagger}\right)|\mathrm{vac}\rangle,
$$

where $|\mathrm{vac}\rangle$ is the vacuum and $c_{j \sigma}^{\dagger}$ is the creation operator of electron at the $j$ th site with the spin $\sigma$ along the space-fixed quantization axis; the parameters $C_{j \sigma}$ 's are usually obtained by solving the eigenvalue problem numerically.

When only the energy minimization is required in the optimization, the optimized solution is the one with constant $\chi$ (for example, $\chi=0$ ). An important point to remember is that, in the numerical calculation by computers, the multi-valued phase factors in Eq. (2) are simply translated into numbers, and the possible sign change in Eq. (3) is lost. Then, the obtained wave function in the form given in Eq. (7) is mistakenly regarded as a single-valued function with $e^{-i \frac{\xi_{j}}{2}} C_{j \uparrow}$ and $e^{i \frac{\xi_{j}}{2}} C_{j \uparrow}$ as singlevalued parameters with a constant factor $e^{-i \frac{\chi_{j}}{2}}$ (which is 1 for $\chi=0$ ). Actually, $e^{ \pm i \frac{\xi_{j}}{2}}$ is multi-valued, thus, the obtained wave function does not satisfy the single-valued condition.

In the following, we explain how to impose the single-valued condition: the information about the multi-valuedness of $\xi$ and $\chi$ is encoded in their winding numbers. The phase shift of $\xi$ and $\chi$ after the transportation along the loop $C_{\ell}$ are $2 \pi w_{\ell}[\xi]$ and $2 \pi w_{\ell}[\chi]$, respectively, where $w[\chi]_{\ell}$ is the winding number of $\chi$ along $C_{\ell}$ given by

$$
w[\chi]_{\ell}=\frac{1}{2 \pi} \oint_{C_{\ell}} \nabla \chi \cdot d \mathbf{r}=\frac{1}{2 \pi} \sum_{i=1}^{N_{\ell}}\left(\chi_{C_{\ell}(i+1)}-\chi_{C_{\ell}(i)}\right) .
$$

Then, if the condition

$$
w_{\ell}[\xi]+w_{\ell}[\chi]=\text { even number, }
$$

is satisfied for any loop $C_{\ell}$, the phase change of $\pm \xi_{j}-\chi_{j}$ is a multiple of $4 \pi$ after the transportation. Then, $e^{i \frac{ \pm \xi_{j}}{2}} e^{-i \frac{\chi_{j}}{2}}$ do not change sign; thus, the wave function in Eq. (7) becomes single-valued. Thus, the single-valued condition is satisfied if we impose the constrains in Eq. (9) in the evaluation of the wave functions.

The introduced variable $\chi$ has singularities within the loops where those of $\xi$ exist. It is a harmonic function that satisfies

$$
\nabla^{2} \chi=0 \text {. }
$$


If the added phase factor $e^{-i \frac{\chi_{j}}{2}}$ in Eq. (6) is transferred from the wave function to the Hamiltonian, the momentum operator $\mathbf{p}$ is replaced as

$$
\mathbf{p} \rightarrow \mathbf{p}-\frac{\hbar}{2} \nabla \chi
$$

This should be compared with the modification of the momentum operator in the electromagnetic field with the vector potential $\mathbf{A}^{\mathrm{em}}$,

$$
\mathbf{p} \rightarrow \mathbf{p}-\frac{q}{c} \mathbf{A}^{\mathrm{em}},
$$

where the superscript 'em' stands for 'electromagnetic' and denotes that it is the electromagnetic field origin.

The comparison of Eqs. (11) and (12) shows that the vector potential

$$
\mathbf{A}^{\mathrm{fic}}=\frac{c \hbar}{2 q} \nabla \chi
$$

is induced in the system, where the charge unit $q$ is given by the electron charge $q=-e$, $c$ is the speed of light, and $\hbar$ is Planck's constant divided by $2 \pi$. Here, the superscript 'fic' in $\mathbf{A}^{\text {fic }}$ stands for 'fictitious' and denotes that it is not the electromagnetic field origin. Since the 'magnetic field' produced by $\mathbf{A}^{\text {fic }}$ is zero, i.e. $\nabla \times \mathbf{A}^{\text {fic }}=0$, the vector potential is of an 'Aharonov-Bohm effect' type [28].

We consider the novel superconductivity that occurs when the ordinary electric current generation by the single-particle excitations are suppressed by an energy gap formation, but the novel current generation by the appearance of the non-trivial $\mathbf{A}^{\text {fic }}$ occurs. We call this superconductivity, spin-vortex superconductivity [15].

When both $\mathbf{A}^{\mathrm{em}}$ and $\mathbf{A}^{\text {fic }}$ exist, the modification of the momentum operator becomes

$$
\mathbf{p} \rightarrow \mathbf{p}-\frac{q}{c} \mathbf{A}^{\mathrm{em}}-\frac{q}{c} \mathbf{A}^{\text {fic }}
$$

Actually, the vector potential $\mathbf{A}^{\mathrm{em}}$ always exists including the case where it is a pure gauge given by $\nabla f$, where $f$ is a single-valued function. When the nontrivial $\mathbf{A}^{\text {fic }}$ appears, the electric current appears; then, $\mathbf{A}^{\mathrm{em}}$ is generated by the electric current. External magnetic fields also give rise to $\mathbf{A}^{\mathrm{em}}$.

The persistent current density is given by the

$$
\mathbf{j}=-c \frac{\delta E\left[\mathbf{A}^{\mathrm{eff}}\right]}{\delta \mathbf{A}^{\mathrm{eff}}},
$$

where $E\left[\mathbf{A}^{\text {eff }}\right]$ is the total energy and $\mathbf{A}^{\text {eff }}$ is the effective vector potential given by

$$
\mathbf{A}^{\mathrm{eff}}=\mathbf{A}^{\mathrm{em}}+\mathbf{A}^{\text {fic }} \text {. }
$$

Since we perform the energy minimization with the constraints given by Eq. (9), we construct the following functional

$$
F\left[\mathbf{A}^{\mathrm{eff}}\right]=E\left[\mathbf{A}^{\mathrm{eff}}\right]+\sum_{\ell=1}^{N_{\text {loop }}} \lambda_{\ell}\left(\oint_{C_{\ell}} \nabla \chi \cdot d \mathbf{r}-2 \pi w_{\ell}\right),
$$

where the second term in the right-hand side is the term arising from the constrains; $\lambda_{\ell}$ is the Lagrange multiplier, $w_{\ell}$ is the winding number of $\chi$ along a loop $C_{\ell}$, and $N_{\text {loop }}$ is 
the number of independent loops, i.e., any loops in the system can be constructed by the addition and scalar multiplication of them.

The optimization with respect to $\nabla \chi$ yields,

$$
\frac{\delta F\left[\mathbf{A}^{\mathrm{eff}}\right]}{\delta \nabla \chi}=0 .
$$

From the above condition, the current density is obtained as

$$
\mathbf{j}(\mathbf{x})=\frac{2 q}{\hbar} \sum_{\ell=1}^{N_{\text {loop }}} \lambda_{\ell} \frac{\delta}{\delta \nabla \chi(\mathbf{x})} \oint_{C_{\ell}} \nabla \chi \cdot d \mathbf{r} .
$$

where Eq. (15) is used. This indicates that current carrying states arise due to the constraint, and given as the sum of $N_{\text {loop }}$ contributions. The current is a collection of loop currents; each of them is a localized loop current around the core of the spinvortex which we call the spin-vortex-induced loop current (SVILC). In Fig. 2, examples of the current patters produced by the current density in Eq. (19) are depicted. A variety of current patters are possible since the constrains in Eq. (9) have flexibility in the combination of $w_{\ell}$ 's. The details of the calculation for the current density given in Eq. (19) will be given in the next section.

When the number of spin-vortices are large, a large variety of constraints that satisfy Eq. (9) are possible; thus, the current density can flexibly fit various boundary conditions. Even the solution with feeding current from outside can be obtained as will be shown later.

The vector potential for the real electromagnetic field $\mathbf{A}^{\mathrm{em}}$ has an arbitrariness within the gauge transformation

$$
\mathbf{A}^{\mathrm{em}} \rightarrow \mathbf{A}^{\mathrm{em}}+\nabla f
$$

where $f$ is a single-valued function.

When we obtain the optimized $\mathbf{A}^{\text {fic }}$, however, this arbitrariness is negated. This is due to the fact that the choice of the gauge in $\mathbf{A}^{\mathrm{em}}$ is absorbed by the shift in $\mathbf{A}^{\text {fic }}$ as

$$
\mathbf{A}^{\text {fic }} \rightarrow \mathbf{A}^{\text {fic }}-\nabla f \text {. }
$$

As a result, the sum of the two, the effective vector potential $\mathbf{A}^{\text {eff }}$ is invariant. This means that the $\mathbf{A}^{\text {eff }}$ is gauge invariant.

\section{Construction of $E\left[\mathbf{A}^{\text {fic }}\right]$}

In this section, we construct $E\left[\mathbf{A}^{\text {fic }}\right]$. When $E\left[\mathbf{A}^{\text {fic }}\right]$ is obtained, we can calculate the persistent current from the stationary condition of the functional in Eq. (17). Although the energy functional $E\left[\mathbf{A}^{\text {fic }}\right]$ will be derived using a number of approximations below, they are not essential for the appearance of $\mathbf{A}^{\text {fic }}$; the only requisite is the presence of 'spin-vortices' or the objects described by $\xi$ with non-zero winding numbers. For the persistent current generation, the nonzero $\mathbf{A}^{\text {fic }}$ dependence of the total energy is also necessary. 

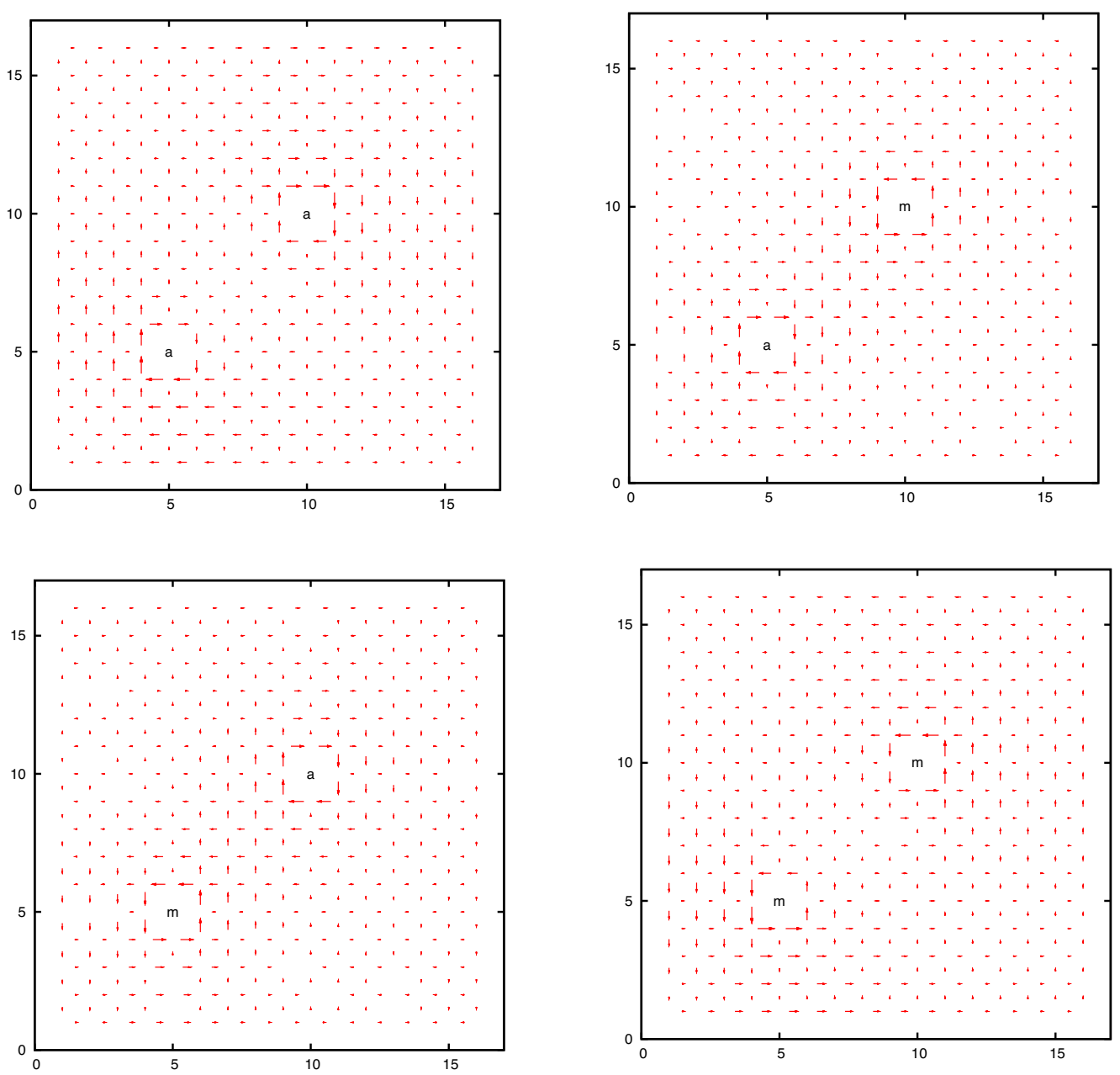

Figure 2. Spin-vortex-induced loop currents in Eq. (19) for the spin configurations in Fig. 1. In the actual calculation the procedure explained in Section 4 is employed. ' $\mathrm{m}$ ' and ' $\mathrm{a}$ ' indicate cores of spin-vortices with winding numbers +1 and -1 in Eq. (8), respectively.

The minimal model for the parent antiferromagnetic insulator of the cuprate is a half-filled 2D Hubbard model with the large on-site Coulomb repulsion. We use the model in which hole-lattice interaction is added to the Hubbard model;

$$
H=-\sum_{i, j, \sigma} t_{i j} c_{i \sigma}^{\dagger} c_{j \sigma}+U \sum_{j} c_{j \uparrow}^{\dagger} c_{j \uparrow} c_{j \downarrow}^{\dagger} c_{j \downarrow}+H_{\text {hole+lattice }}
$$

where $t_{i j}$ is $t$ when the sites $i$ and $j$ are nearest neighbors, and zero otherwise. The strong correlation condition, $0<t \ll U$, is assumed. The third term describes the interaction between holes and underlying lattice, and also lattice vibrations.

The Hamiltonian $H$ can be simplified if we utilize the following observations: 1 ) the Hall coefficient measurement in the sample where the superconducting state is destroyed by a strong magnetic field indicates that the charge carriers are electrons [32]; 2) a molecular orbital cluster calculation indicates that the energy lowering by the deformation of $\mathrm{CuO}$ unit is large enough to localize the hole [21]. 
The above observations indicates that the holes become small polarons and immobile at low temperatures in the bulk. Thus, we may remove the hole-occupied sites as inaccessible sites for electrons; then, the system is in the 'effectively-half-filled situation' (EHFS), where the number of accessible sites for conduction electrons and that of the conduction electrons are equal.

In order to describe the wave function for the conduction electron in the selfconsistent field with the spin-vortices, the following new annihilation operators are introduced;

$$
\left(\begin{array}{c}
a_{j} \\
b_{j}
\end{array}\right)=\frac{e^{i \frac{\chi_{j}}{2}}}{\sqrt{2}}\left(\begin{array}{cc}
e^{i \frac{\xi_{j}}{2}} & e^{-i \frac{\xi_{j}}{2}} \\
-e^{i \frac{\xi_{j}}{2}} & e^{-i \frac{\xi_{j}}{2}}
\end{array}\right)\left(\begin{array}{c}
c_{j \uparrow} \\
c_{j \downarrow}
\end{array}\right) .
$$

The spin-vortices are lying in the conduction plane (the $\mathrm{CuO}_{2}$ plane), and the angular variable $\xi_{j}$ is identified as the spin direction at site $j$ in the conduction plane. The variable $\chi_{j}$ is necessary for the single-valuedness of the above transformation matrix; since $\xi$ is an angular variable with $\xi_{j}$ and $\xi_{j}+2 \pi$ physically equivalent, the sign-change of $e^{ \pm \frac{i}{2} \xi_{j}}$ by the phase shift $\xi_{j} \rightarrow \xi_{j}+2 \pi$ must be compensated by the sign-change of $e^{\frac{i}{2} \chi_{j}}$. Actually, the phase factor $e^{i \frac{\chi_{j}}{2}}$ in Eq. (23) gives rise to the phase factor in Eq. (6).

After the basis transformation, the Hamiltonian for the EHFS $H_{\text {EHFS }}$ is given by

$$
\begin{aligned}
H_{\mathrm{EHFS}}\left[\mathbf{A}^{\mathrm{fic}}\right] & =-\sum_{k, j \in \text { acc. sites }} t_{k j} e^{\frac{i}{2} \int_{j}^{k} \nabla \chi \cdot d \mathbf{r}}\left[\cos \frac{\xi_{k}-\xi_{j}}{2}\left(a_{k}^{\dagger} a_{j}+b_{k}^{\dagger} b_{j}\right)-i \sin \frac{\xi_{k}-\xi_{j}}{2}\left(a_{k}^{\dagger} b_{j}+b_{k}^{\dagger} a_{j}\right)\right] \\
& +U \sum_{j \in \text { acc. sites }} a_{j}^{\dagger} a_{j} b_{j}^{\dagger} b_{j}
\end{aligned}
$$

where $H_{\text {hole+lattice }}$ is omitted by taking into account of its effect as the inaccessible sites formation. A notable point in Eq. (24) is that the transfer integrals acquire phase factor $e^{\frac{i}{2} \int_{j}^{k} \nabla \chi \cdot d \mathbf{r}}$. This factor is the one that will appear if the Peierls substitution for a magnetic field with the vector potential $\mathbf{A}^{\text {fic }}=\frac{c \hbar}{2 q} \nabla \chi$ is performed.

To obtain the ground state wave function, we employ a mean field approximation since a single Slater determinant description is expected to be a good one due to a large energy gap between the occupied levels and unoccupied levels in the EHFS. The mean field approximation further simplifies the Hamiltonian as

$$
\begin{aligned}
\bar{H}_{\mathrm{EHFS}}\left[\mathbf{A}^{\mathrm{fic}}\right] & =-\sum_{k, j \in \text { acc. sites }} t_{k j} e^{\frac{i}{2} \int_{j}^{k} \nabla \chi \cdot d \mathbf{r}}\left[\cos \frac{\xi_{k}-\xi_{j}}{2}\left(a_{k}^{\dagger} a_{j}+b_{k}^{\dagger} b_{j}\right)-i \sin \frac{\xi_{k}-\xi_{j}}{2}\left(a_{k}^{\dagger} b_{j}+b_{k}^{\dagger} a_{j}\right)\right] \\
& +U \sum_{j \in \text { acc. sites }}\left(\left\langle a_{j}^{\dagger} a_{j}\right\rangle b_{j}^{\dagger} b_{j}+\left\langle b_{j}^{\dagger} b_{j}\right\rangle a_{j}^{\dagger} a_{j}\right) .
\end{aligned}
$$

From the above Hamiltonian, the total energy $E\left[\mathbf{A}^{\text {fic }}\right]$ is obtained.

$E\left[\mathbf{A}^{\text {eff }}\right]$ in the functional in Eq. (17) is obtained by replacing $\mathbf{A}^{\text {fic }}$ by $\mathbf{A}^{\text {eff }}$. In a small system without an external field, however, the effect of $\mathbf{A}^{\mathrm{em}}$ is negligibly small compared with $\mathbf{A}^{\text {fic }}$, thus, it may be omitted.

The current obtained here flows without external fields in the situation where the lower energy band is filled and the upper energy band is empty. Usually, the sum of the current contributions from all electrons in the filled band is zero; thus, it is usually 
believed that the filled band does not contribute to the electric current. However, in the present case, due to the existence of the internal field $\mathbf{A}^{\text {fic }}$, the sum of the current contributions from all electrons in the filled band becomes nonzero; thus, the current is not zero. The current produced by $\mathbf{A}^{\text {fic }}$ gives rise to $\mathbf{A}^{\mathrm{em}}$, thus, the total gauge potential is the sum of the two, $\mathbf{A}^{\mathrm{eff}}=\mathbf{A}^{\mathrm{fic}}+\mathbf{A}^{\mathrm{em}}$. In other words, the appearance of $\mathbf{A}^{\text {fic }}$ makes the vector potential in the system $\mathbf{A}^{\text {eff }}$ instead of $\mathbf{A}^{\mathrm{em}}$.

Since we have obtained the electronic state of the filled lower band, its total wave function is the Slater-determinant of the conduction electron wave functions of the filled band. Actually, Slater-determinant states with many different current patters are possible within the constraints in Eq. (9). Linear combinations of them also satisfy the single-valued condition of the wave function. In the following, however, we only consider the situation where the system is described by a single Slater determinant.

Now, let us summarize the flow of calculation procedures: first, we obtain $\xi_{k}-\xi_{j}$ using the classical spin Hamiltonian in Eq. (1). For this step, we introduce an angle variable $\eta$ give by

$$
\xi_{j}=\pi\left(j_{x}+j_{y}\right)+\eta_{j}
$$

where $j=\left(j_{x}, j_{y}\right)$ is the $j$ th site in the two-dimensional square lattice and the first term describes the antiferromagnetic background. Then, $\eta$ describes the spin-vortices; the winding number for $\xi$ is actually calculated with $\eta$; the difference of $\eta$ between nearby sites is in the range,

$$
-\pi \leq \eta_{\ell}-\eta_{k}<\pi .
$$

At this point, the multi-valuedness of $\xi$ is not taken into account; we just calculate $\xi_{k}-\xi_{j}$ and solve the Hartree-Fock equations using Eq. (25) with $\chi=0$. The $\alpha$ th wave function obtained is given by

$$
|\tilde{\alpha}\rangle=\sum_{j}\left(A_{j}^{\alpha} \tilde{a}_{j}^{\dagger}+B_{j}^{\alpha} \tilde{b}_{j}^{\dagger}\right)|\operatorname{vac}\rangle
$$

where $A_{j}^{\alpha}$ and $B_{j}^{\alpha}$ are numerically obtained; $\tilde{a}_{j}^{\dagger}$ and $\tilde{b}_{j}^{\dagger}$ are given through the relations,

$$
\left(\begin{array}{c}
\tilde{a}_{j} \\
\tilde{b}_{j}
\end{array}\right)=\frac{1}{\sqrt{2}}\left(\begin{array}{cc}
e^{i \frac{\xi_{j}}{2}} & e^{-i \frac{\xi_{j}}{2}} \\
-e^{i \frac{\xi_{j}}{2}} & e^{-i \frac{\xi_{j}}{2}}
\end{array}\right)\left(\begin{array}{c}
c_{j \uparrow} \\
c_{j \downarrow}
\end{array}\right) .
$$

since we put $\chi=0$. At this point, the multi-valuedness of $\xi$, or jumps of its value by $2 \pi n$, where $n$ is an integer, are neglected.

To impose single-valued condition with including the multi-valuedness of $\xi$, we replace $\tilde{a}_{j}^{\dagger}$ and $\tilde{b}_{j}^{\dagger}$ by $a_{j}^{\dagger}$ and $b_{j}^{\dagger}$,

$$
|\alpha\rangle=\sum_{j}\left(A_{j}^{\alpha} a_{j}^{\dagger}+B_{j}^{\alpha} b_{j}^{\dagger}\right)|\mathrm{vac}\rangle .
$$

Using $\{|\alpha\rangle\}, E\left[\mathbf{A}^{\text {fic }}\right]$ is obtained from Eq. (25). Then, $\nabla \chi$ is obtained from the stationary condition for $F\left[\mathbf{A}^{\text {fic }}\right]$.

If $|\alpha\rangle$ is expressed with $c_{j \uparrow}^{\dagger}$ and $c_{j \downarrow}^{\dagger}$, it is given by

$$
|\alpha\rangle=\sum_{j}\left(e^{-i \frac{\xi_{j}+\chi_{j}}{2}} \frac{A_{j}^{\alpha}-B_{j}^{\alpha}}{\sqrt{2}} c_{j \uparrow}^{\dagger}+e^{i \frac{\xi_{j}-\chi_{j}}{2}} \frac{A_{j}^{\alpha}+B_{j}^{\alpha}}{\sqrt{2}} c_{j \downarrow}^{\dagger}\right)|\mathrm{vac}\rangle,
$$


where the values of $\xi$ and $\chi$ are obtained by taking into account their multi-valuedness.

From the optimization of the functional given in Eq. (17), values of $\left(\chi_{\ell}-\chi_{k}\right)$ where $\ell$ and $k$ are connected by the transfer integral $t_{\ell k}$ are obtained. The evaluation of $\xi_{j}-\chi_{j}$ and $\xi_{j}+\chi_{j}$ must be done in a path integral manner; they are evaluated along a path starting from an initial point with initial values. The path may have branches.

Then, $\left(\chi_{\ell}-\chi_{k}\right)$ are obtained by the stationary condition of the functional given in Eq. (17), where $w_{\ell}$ must be supplied to satisfy the condition in Eq. (9). Different combinations of $w_{\ell}$ 's yield different current patterns. The difference of $\chi$ between nearby sites is in the range,

$$
-\pi \leq \chi_{\ell}-\chi_{k}<\pi
$$

Values of $\eta$ and $\chi$ are rebuilt from the values $\eta_{\ell}-\eta_{k}$ and $\chi_{\ell}-\chi_{k}$, respectively; the step where values of $\eta_{\ell}$ and $\chi_{\ell}$ are derived from the already evaluated values of $\eta_{k}$ and $\chi_{k}$ is given by

$$
\begin{aligned}
& \eta_{\ell}=\eta_{k}+\left(\eta_{\ell}-\eta_{k}\right) \\
& \chi_{\ell}=\chi_{k}+\left(\chi_{\ell}-\chi_{k}\right)
\end{aligned}
$$

where the sites $\ell$ and $k$ are connected by a bond in the path. From $\eta_{j}$, the value of $\xi_{j}$ is obtained using the relation in Eq. (26). This process is continued until values at all accessible sites are evaluated once and only once. Using $\xi_{j}$ and $\chi_{j}$ obtained from the above procedure, we obtain values of $\xi_{j}-\chi_{j}$ and $\xi_{j}+\chi_{j}$.

Since the values of $\xi$ and $\chi$ are path-dependent they have $2 \pi n$ ( $n$ is an integer) jumps between sites that are connected by bonds but not used during the evaluation process described above. Due to the condition in Eq. (9), the phase jumps for $\xi \pm \chi$ are $4 \pi n$ ( $n$ is an integer). Then, values of $e^{-i \frac{\xi_{j}+\chi_{j}}{2}}$ and $e^{i \frac{\xi_{j}-\chi_{j}}{2}}$ are path-independent because the $4 \pi n$ jumps are absorbed by $e^{i 2 \pi n}=1$. In this way, the single-valued wave functions $\{|\alpha\rangle\}$ are obtained.

\section{An example}

For definiteness, we work out persistent current calculations described in the previous section using the model system of a $4 \times 3$ lattice depicted in Fig. 3 .

There are three independent loops $C_{1}, C_{2}$, and $C_{3}$ as seen in Fig. 4; other loops are constructed from them; thus, the constrains in Eq. (9) are imposed for those three loops.

The functional $F$ in Eq. (17) for this system is given by

$$
\begin{aligned}
& F_{\mathrm{EHFS}}\left[\tau_{1}, \cdots, \tau_{7}, \tau_{a}, \cdots, \tau_{f}, \lambda_{1}, \lambda_{2}, \lambda_{3}\right] \\
= & E_{\mathrm{EHFS}}\left[\frac{c \hbar}{2 q} \tau_{1}, \cdots, \frac{c \hbar}{2 q} \tau_{f}\right]+\frac{\lambda_{1}}{2 \pi}\left(\tau_{5}+\tau_{6}+\tau_{e}+\tau_{b}-\tau_{2}-\tau_{1}-\tau_{a}-\tau_{d}-2 \pi w_{1}\right) \\
+ & \frac{\lambda_{2}}{2 \pi}\left(\tau_{4}+\tau_{c}-\tau_{3}-\tau_{b}\right)+\frac{\lambda_{3}}{2 \pi}\left(\tau_{7}+\tau_{f}-\tau_{4}-\tau_{e}\right),
\end{aligned}
$$

where $\tau_{j}$ 's are differences of the phase $\chi$ and $\lambda_{k}$ 's are the Lagrange multipliers introduced to impose the constraints in Eq. (9); their definitions are given in Fig. 4. 
(b)

(a)
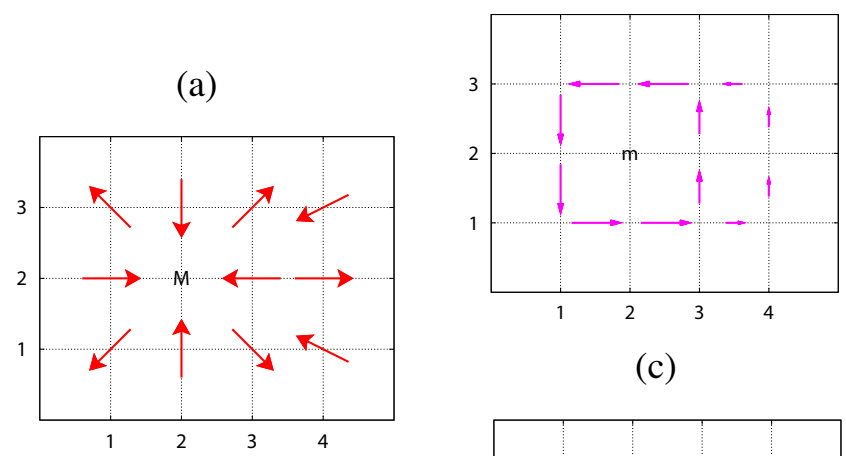

(c)

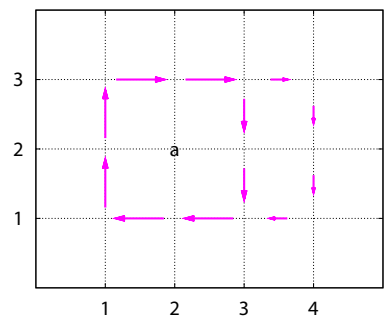

Figure 3. (a) spin configuration with one spin-vortex with winding number +1 in the antiferromagnetic background; (b) loop current with winding number +1 ; (c) loop current with winding number -1 .

Currents $J_{k}$ for bonds $k$ can be calculated using the following formula,

$$
J_{k}=-\frac{2 q}{\hbar} \frac{\partial E_{\mathrm{EHFS}}}{\partial \tau_{k}},
$$

which corresponds to Eq. (15). Then, stationary conditions of the function $F_{\text {EHFs }}$ with respect to $\tau$ 's yield relations corresponding to Eq. (19);

$$
\begin{aligned}
& J_{1}=\frac{2 q}{\hbar} \lambda_{1}, \quad J_{2}=\frac{2 q}{\hbar} \lambda_{1}, \quad J_{3}=\frac{2 q}{\hbar} \lambda_{2} \\
& J_{4}=\frac{2 q}{\hbar}\left(\lambda_{3}-\lambda_{2}\right), \quad J_{5}=-\frac{2 q}{\hbar} \lambda_{1}, \quad J_{6}=-\frac{2 q}{\hbar} \lambda_{1} \\
& J_{7}=-\frac{2 q}{\hbar} \lambda_{3}, \quad J_{a}=\frac{2 q}{\hbar} \lambda_{1}, \quad J_{b}=\frac{2 q}{\hbar}\left(\lambda_{2}-\lambda_{1}\right) \\
& J_{c}=-\frac{2 q}{\hbar} \lambda_{2}, \quad J_{d}=\frac{2 q}{\hbar} \lambda_{1}, \quad J_{e}=\frac{2 q}{\hbar}\left(\lambda_{3}-\lambda_{1}\right) \\
& J_{f}=-\frac{2 q}{\hbar} \lambda_{3}
\end{aligned}
$$

We approximate $J_{k}$ to be linear in $\tau_{k}$ as

$$
J_{k}=-\frac{2 q}{\hbar} \frac{\partial^{2} E_{\mathrm{EHFS}}}{\partial \tau_{k}^{2}} \tau_{k}
$$

which is a good approximation if $\tau_{k}$ is so small that can be approximated as $\sin \frac{\tau_{k}}{2} \approx \frac{\tau_{k}}{2}$.

Then, the constraints with respect to the winding numbers yield the following linear equations for $\lambda_{1}, \lambda_{2}$, and $\lambda_{3}$;

$$
\left(\begin{array}{l}
2 \pi w_{1} \\
2 \pi w_{2} \\
2 \pi w_{3}
\end{array}\right)=M\left(\begin{array}{c}
\lambda_{1} \\
\lambda_{2} \\
\lambda_{3}
\end{array}\right),
$$






Figure 4. A $4 \times 3$ lattice with one hole-occupied site. The hole occupies $(1,1)$. There are three independent loops: $C_{1}$ is a loop that connects $(0,0) \rightarrow(2,0) \rightarrow(2,2) \rightarrow$ $(0,2) \rightarrow(0,0) ; C_{2}$ is a loop that connects $(2,1) \rightarrow(3,1) \rightarrow(3,2) \rightarrow(2,2) \rightarrow(2,1)$; $C_{3}$ is a loop that connects $(2,0) \rightarrow(3,0) \rightarrow(3,1) \rightarrow(2,1) \rightarrow(2,0)$. $\tau$ indicates a difference of $\chi$ values at two sites; for example, $\tau_{1}=\chi(1,2)-\chi(0,2)$.

where the matrix $M$ is given by

$$
M=\left(\begin{array}{ccc}
\frac{1}{D_{1}}+\frac{1}{D_{2}}+\frac{1}{D_{b}}+\frac{1}{D_{e}}+\frac{1}{D_{6}}+\frac{1}{D_{5}}+\frac{1}{D_{d}}+\frac{1}{D_{a}} & -\frac{1}{D_{b}} & -\frac{1}{D_{e}} \\
-\frac{1}{D_{b}} & \frac{1}{D_{3}}+\frac{1}{D_{c}}+\frac{1}{D_{4}}+\frac{1}{D_{b}} & -\frac{1}{D_{4}} \\
-\frac{1}{D_{e}} & -\frac{1}{D_{4}} & \frac{1}{D_{4}}+\frac{1}{D_{f}}+\frac{1}{D_{7}}+\frac{1}{D_{e}}
\end{array}\right)
$$

and

$$
D_{k}=\frac{\partial^{2} E_{\mathrm{EHFS}}}{\partial \tau_{k}^{2}}
$$

Then, from $\lambda$ 's, we calculate the currents using Eq. (36). The results are depicted in Fig. 3. A similar calculation yields the current in Fig. 2.

Note that the current obtained by optimizing $\tau$ 's satisfies the conservation of charge since it is made as a sum of loop currents.

Let us now consider the inclusion of real magnetic fields. It is taken into account by replacing the phase factor in the hopping term as

$$
e^{\frac{i q}{c \hbar} \int_{j}^{k} \mathbf{A}^{\mathrm{fic}} \cdot d \mathbf{r}} \rightarrow e^{\frac{i q}{c \hbar} \int_{j}^{k}\left(\mathbf{A}^{\mathrm{em}}+\mathbf{A}^{\mathrm{fic}}\right) \cdot d \mathbf{r}} .
$$

This causes the replacement

$$
E\left[\mathbf{A}^{\text {fic }}\right] \rightarrow E\left[\mathbf{A}^{\text {fic }}+\mathbf{A}^{\mathrm{em}}\right]
$$

We approximate $J_{k}$ to be linear in $\mathbf{A}^{\text {eff }}$ as

$$
J_{k}=-\frac{2 q}{\hbar} \frac{\partial^{2} E_{\mathrm{EHFS}}}{\partial \tau_{k}^{2}}\left(\tau_{k}+\alpha_{k}\right)
$$

where, for example, $\alpha_{1}$ is defined as

$$
\alpha_{1}=\frac{q}{\hbar c} \int_{(0,2)}^{(1,2)} \mathbf{A}^{\mathrm{em}} \cdot d \mathbf{r} .
$$

Then, replacing $J_{k}$ in Eq. (36) by that in Eq. (43) the equations for $\lambda$ 's become

$$
\left(\begin{array}{c}
2 \pi w_{1}+\alpha_{1}+\alpha_{2}-\alpha_{b}-\alpha_{e}-\alpha_{6}-\alpha_{5}+\alpha_{d}+\alpha_{a} \\
2 \pi w_{2}+\alpha_{3}-\alpha_{c}-\alpha_{4}+\alpha_{b} \\
2 \pi w_{3}+\alpha_{4}-\alpha_{f}-\alpha_{7}+\alpha_{e}
\end{array}\right)=M\left(\begin{array}{c}
\lambda_{1} \\
\lambda_{2} \\
\lambda_{3}
\end{array}\right)
$$






Figure 5. The same $3 \times 4$ lattice as in Fig. 4 but current $J_{\text {ex }}$ is fed to $(0,0)$ and extracted from $(3,2)$.

The current in Eq. (43) indicates that the Meissner effect will occur if the system is sufficiently large, since the diamagnetic current proportional to $\mathbf{A}^{\mathrm{em}}$ flows.

Now let us consider the case where a current is fed from outside. The current feeding is included in the following manner: let us examine the system depicted in Fig. 5. We include the hopping term between $(0,0)$ and $(3,2)$ and introduce a loop $C_{4}$ that connects $(0,0) \rightarrow(2,0) \rightarrow(3,0) \rightarrow(3,1) \rightarrow(3,2) \rightarrow(0,0)$; the Lagrangian multiplier for this loop is $\lambda_{4}$

Then, the equations for $\lambda_{1}, \lambda_{2}$, and $\lambda_{3}$ are obtained as

$$
\left(\begin{array}{c}
2 \pi w_{1}+\left(\frac{1}{D_{5}}+\frac{1}{D_{6}}\right) \lambda_{4} \\
2 \pi w_{2}+\frac{1}{D_{c}} \lambda_{4} \\
2 \pi w_{3}+\left(\frac{1}{D_{7}}+\frac{1}{D_{f}}\right) \lambda_{4}
\end{array}\right)=M\left(\begin{array}{c}
\lambda_{1} \\
\lambda_{2} \\
\lambda_{3}
\end{array}\right) .
$$

The parameter $\lambda_{4}$ is treated as a parameter for the external current; i.e., we relates the external current $J_{\text {ex }}$ to $\lambda_{4}$ as

$$
J_{\text {ex }}=\frac{2 q}{\hbar} \lambda_{4}
$$

where the contributions from $\mathbf{A}^{\mathrm{em}}$ are omitted. We may choose other loop for $C_{4}$ to include $J_{\text {ex }}$; however, the current distribution is unaffected by the choice of the added loop.

\section{Connection between $A^{\text {fic }}$ and London's superpotential}

Let us calculate single-particle wave functions from Eq. (31). We define $\langle\mathbf{r}|$ as

$$
\langle\mathbf{r}|=\langle\operatorname{vac}| \sum_{j}\left[c_{j \uparrow} w_{j \uparrow}(\mathbf{r})+c_{j \downarrow} w_{j \downarrow}(\mathbf{r})\right] .
$$

Then, the single-particle wave function is given by

$$
\begin{aligned}
\langle\mathbf{r} \mid \alpha\rangle & =\sum_{j}\left(e^{-i \frac{\xi_{j}+\chi_{j}}{2}} \frac{A_{j}^{\alpha}-B_{j}^{\alpha}}{\sqrt{2}} w_{j \uparrow}(\mathbf{r})+e^{i \frac{\xi_{j}-\chi_{j}}{2}} \frac{A_{j}^{\alpha}+B_{j}^{\alpha}}{\sqrt{2}} w_{j \downarrow}(\mathbf{r})\right) \\
& \approx \sum_{j}\left(e^{-i \frac{\xi_{j}+\chi(\mathbf{r})}{2}} \frac{A_{j}^{\alpha}-B_{j}^{\alpha}}{\sqrt{2}} w_{j \uparrow}(\mathbf{r})+e^{i \frac{\xi_{j}-\chi(\mathbf{r})}{2}} \frac{A_{j}^{\alpha}+B_{j}^{\alpha}}{\sqrt{2}} w_{j \downarrow}(\mathbf{r})\right)
\end{aligned}
$$




$$
=e^{-\frac{i}{2} \chi(\mathbf{r})}\langle\mathbf{r} \mid \bar{\alpha}\rangle
$$

where $w_{j \sigma}(\mathbf{r})$ is the Wannier function at the $j$ th site with spin $\sigma$, and

$$
\langle\mathbf{r} \mid \bar{\alpha}\rangle=\sum_{j}\left(e^{-i \frac{\xi_{j}}{2}} \frac{A_{j}^{\alpha}-B_{j}^{\alpha}}{\sqrt{2}} w_{j \uparrow}(\mathbf{r})+e^{i \frac{\xi_{j}}{2}} \frac{A_{j}^{\alpha}+B_{j}^{\alpha}}{\sqrt{2}} w_{j \downarrow}(\mathbf{r})\right) .
$$

In the process of the replacement of $e^{-i \frac{\chi_{j}}{2}} w_{j \sigma}(\mathbf{r})$ by $e^{-i \frac{\chi(\mathbf{r})}{2}} w_{j \sigma}(\mathbf{r})$ in Eq. (49), the fact is used that $w_{j \sigma}(\mathbf{r})$ has significant amplitude only near the $j$ th site.

Then, the total wave function is give by

$$
\begin{aligned}
\Psi\left(\mathbf{r}_{1}, \cdots, \mathbf{r}_{N} ; \xi, \chi\right) & =\frac{1}{\sqrt{N !} \mid}\left|\begin{array}{cccc}
\left\langle\mathbf{r}_{1} \mid 1\right\rangle & \left\langle\mathbf{r}_{2} \mid 1\right\rangle & \cdots & \left\langle\mathbf{r}_{N} \mid 1\right\rangle \\
\left\langle\mathbf{r}_{1} \mid 2\right\rangle & \left\langle\mathbf{r}_{2} \mid 2\right\rangle & \cdots & \left\langle\mathbf{r}_{N} \mid 2\right\rangle \\
\cdot & \cdot & \cdots & \cdot \\
\cdot & \cdot & \cdots & \cdot \\
\left\langle\mathbf{r}_{1} \mid N\right\rangle & \left\langle\mathbf{r}_{2} \mid N\right\rangle & \cdots & \left\langle\mathbf{r}_{N} \mid N\right\rangle
\end{array}\right| \\
& \approx \frac{e^{-\frac{i}{2} \sum_{j} \chi\left(\mathbf{r}_{j}\right)}}{\sqrt{N !}}\left|\begin{array}{cccc}
\left\langle\mathbf{r}_{1} \mid \overline{1}\right\rangle & \left\langle\mathbf{r}_{2} \mid \overline{1}\right\rangle & \cdots & \left\langle\mathbf{r}_{N} \mid \overline{1}\right\rangle \\
\left\langle\mathbf{r}_{1} \mid \overline{2}\right\rangle & \left\langle\mathbf{r}_{2} \mid \overline{2}\right\rangle & \cdots & \left\langle\mathbf{r}_{N} \mid \overline{2}\right\rangle \\
\cdot & \cdot & \cdots & \cdot \\
\cdot & \cdot & \cdots & \cdot \\
\left\langle\mathbf{r}_{1} \mid \bar{N}\right\rangle & \left\langle\mathbf{r}_{2} \mid \bar{N}\right\rangle & \cdots & \left\langle\mathbf{r}_{N} \mid \bar{N}\right\rangle
\end{array}\right| \\
& =e^{-\frac{i}{2} \sum_{j} \chi\left(\mathbf{r}_{j}\right)} \Psi\left(\mathbf{r}_{1}, \cdots, \mathbf{r}_{N} ; \xi, 0\right) .
\end{aligned}
$$

The above wave function has the form suggested by London for superconductors [33]. Actually, Landau also suggested the same wave function form [34].

London argued that the superconducting state is characterized by the long range order of the momentum $\mathbf{p}_{s}$ given by

$$
\mathbf{p}_{s}=\nabla \chi_{s}
$$

and called, $\chi_{s}$, the superpotential; the wave function is expressed as

$$
\Psi_{\mathrm{L}}\left(\mathbf{r}_{1}, \cdots, \mathbf{r}_{N}\right)=e^{\frac{i}{\hbar} \sum_{j} \chi_{s}\left(\mathbf{r}_{j}\right)} \Psi_{0}\left(\mathbf{r}_{1}, \cdots, \mathbf{r}_{N}\right),
$$

using $\chi_{s}[33]$.

The comparison of Eqs. (51) and (53) indicates that we can identify

$$
\chi_{s}=-\frac{\hbar}{2} \chi
$$

Thus, we have

$$
\mathbf{p}_{s}=-\frac{q}{c} \mathbf{A}^{\text {fic }} .
$$

This shows that $\mathbf{A}^{\text {fic }}$ is essentially the long range momentum order envisaged by London [33]. In the spin-vortex superconductivity theory, the existence of $\mathbf{A}^{\text {fic }}$ is the characteristic of superconductors. Thus, the London's explanation of superconductivity and the spin-vortex superconductivity coincide.

Let us calculate the current density; from Eq. (51), we have

$$
E=\left\langle\Psi[\chi]\left|H\left[\mathbf{A}^{\mathrm{em}}\right]\right| \Psi[\chi]\right\rangle=\left\langle\Psi[0]\left|H\left[\mathbf{A}^{\mathrm{em}}+\mathbf{A}^{\mathrm{fic}}\right]\right| \Psi[0]\right\rangle,
$$


thus, the current density is given by

$$
\mathbf{j}=-c\left\langle\Psi[0]\left|\frac{\delta H\left[\mathbf{A}^{\mathrm{em}}+\mathbf{A}^{\mathrm{fic}}\right]}{\delta \mathbf{A}^{\mathrm{em}}}\right| \Psi[0]\right\rangle .
$$

If the kinetic energy is expressed as

$$
K=\sum_{j} \frac{1}{2 m}\left(\mathbf{p}_{j}-\frac{q}{c} \mathbf{A}^{\mathrm{em}}\left(\mathbf{r}_{j}\right)\right)^{2},
$$

where $m$ is the effective mass of electron, the current density for $\Psi\left(\mathbf{r}_{1}, \cdots, \mathbf{r}_{N} ; \xi, \chi\right)$ is calculated as

$$
\mathbf{j}=-\frac{q^{2} \rho}{m c}\left(\mathbf{A}^{\mathrm{em}}+\mathbf{A}^{\mathrm{fic}}\right)=-\frac{q^{2} \rho}{m c} \mathbf{A}^{\mathrm{eff}},
$$

where $\rho$ is the electron density and $\Psi\left(\mathbf{r}_{1}, \cdots, \mathbf{r}_{N} ; \xi, 0\right)$ is assumed to be currentless. This is the gauge invariant current since $\mathbf{A}^{\text {eff }}$ is gauge invariant. The above current expression is obtained by taking

$$
\Psi_{\text {order }}=\rho^{1 / 2} e^{-i \frac{1}{2} \chi}
$$

as the order parameter.

\section{Discussion}

The new criterion for the occurrence of superconductivity put forward by the spin-vortex superconductivity is the appearance of the stable $\mathbf{A}^{\text {fic }}$, which is the gauge potential induced by the single-valued wave function constraint in the presence of the spinvortices. The occurrence of the superconductivity in the cuprate may be judged by this new criterion. The current element is the spin-vortex-induced loop current (SVILC). Since a single SVILC is rather localized as is shown in Fig. 2, the generation of a macroscopic persistent current requires a certain density of spin-vortices; this will explain the existence of the lower limit for the doping concentration (about $x=0.05$ ) in the occurrence of superconductivity in the cuprate.

In the cuprate, the spin-vortices are expected to be particularly stable; one reason is that the doped holes provide with their cores. In addition, the spin exchange between spins across the hole occupied sites may stabilize the spin-vortices in the following way: the classical spin Hamiltonian for the hole-doped cuprate with the spin exchange between spins across the hole occupied sites is given by

$$
E[\xi]=\frac{J}{4} \sum_{\langle i, j\rangle_{1} ; i, j \in \text { acc. sites }} \cos \left(\xi_{i}-\xi_{j}\right)+\frac{J^{\prime}}{4} \sum_{\langle i, j\rangle_{h} ; i, j \in \text { acc. sites }} \cos \left(\xi_{i}-\xi_{j}\right),
$$

where $\langle i, j\rangle_{h}$ indicates the paris across the hole occupied sites; it includes also pairs of sites for which the sites $i$ and $j$ are in the right angle positions with respect to the hole occupied site. Then, the quartet of the spin-vortices of the $4 a \times 4 a$ size, where $a$ is the lattice constant in the $\mathrm{CuO}_{2}$ plane, depicted in Fig. 6 becomes energetically stable if the condition $J^{\prime}>0.24 J$ is satisfied.

Actually, a large $J^{\prime}$ value is not unlikely in the cuprate due to the small polaron formation: the molecular orbital cluster calculation result indicates that when the small 
(a)

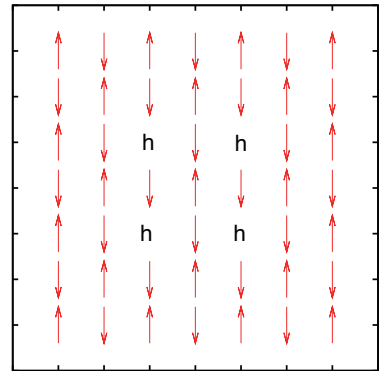

(c)

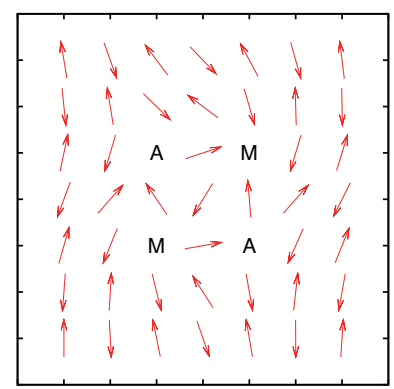

(b)

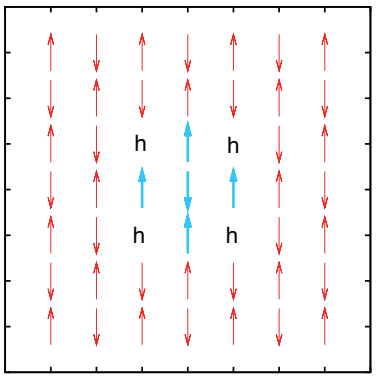

(d)

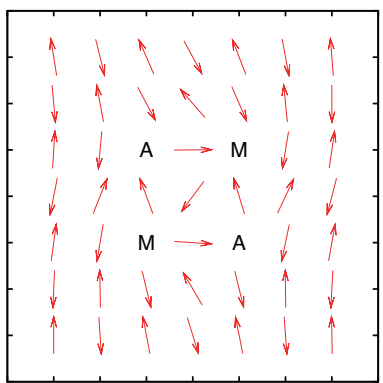

Figure 6. (a) A spin configuration with four holes in the antiferromagnetic background. ' $h$ ' indicates the hole occupied site. (b) A spin configuration with four holes in the antiferromagnetic background but five spins are reversed. If $J^{\prime}>0.25 J$, the spin configuration in (b) has less energy than that in (a). (c) The optimized spin configuration with four spin-vortices with their centers at the hole occupied sites. $J=1$ and $J^{\prime}=0.25$ are used in Eq. (61). ' $\mathrm{M}$ 'and 'A' indicate cores of spin-vortices with winding numbers +1 and -1 in Eq. (4), respectively. The numerical calculation indicates that the configuration with the quartet of spin-vortices like that in (c) has less energy than the antiferromagnetic one down to $J^{\prime}=0.24 J$. (d) The optimized spin configuration obtained by the steepest-decent algorithm starting from the configuration in (c) with $J=1$ and $J^{\prime}=0$.

polaron is formed, $d_{x^{2}-y^{2}}$ and the surrounding four $p_{\sigma}$ orbitals form a molecular orbital [21]. Then, the exchange parameter $J^{\prime}$ across the hole occupied sites is calculated by treating the hole molecular orbital as the intermediate level for the perturbation calculation (Fig. 7) [35];

$$
J^{\prime} \approx \frac{4 t_{d h}^{4}}{\left(\varepsilon_{h}-\varepsilon_{d}\right)^{3}},
$$

where the parameter $t_{d h}$ is the transfer integral between the spin-reside copper $d_{x^{2}-y^{2}}$ orbital and the hole orbital $h$ at the hole-occupied site; $\varepsilon_{d}$ and $\varepsilon_{h}$ are the orbital energies of $d_{x^{2}-y^{2}}$ and $h$, respectively. If the hopping integral $t_{d h}$ is not so much different from that between the copper $d_{x^{2}-y^{2}}$ orbital and the nearby oxygen $p_{\sigma}$ orbital, and the energy difference $\varepsilon_{h}-\varepsilon_{d}$ is sufficiently small, the condition $J^{\prime}>0.24 J$ may be achieved.

The argument of the stability of spin-vortices based on the formation of the quartet of the spin-vortices is in accordance with the fact that the superconducting phase 




Figure 7. The energy level diagram for the calculation of $J^{\prime}$ in Eq. (61). $d_{x^{2}-y^{2}}$ is atomic orbital for $\mathrm{Cu}$. $h$ denotes a molecular orbital made of one $d_{x^{2}-y^{2}}$ and surrounding four $p_{\sigma}$ orbitals. It is formed when the $\mathrm{O}-\mathrm{Cu}$ bond length is shortened by the hole doping. See Ref. [21], Fig. 4(d) for its shape.

disappears at $x=0.25$ since beyond this hole concentration, the destruction of the quartets is inevitable. Then, the observed isotope effect of $T_{c}$ may be explained as the isotope effect on the stability of the quartet of the spin-vortcies $[19,18]$.

In conclusion, by using the functional that is the sum of the energy functional and the single-valued constraint, we have clarified that the single-valued condition on the conduction electron wave functions induces a vector potential $\mathbf{A}^{\text {fic }}$ and generates the persistent current if the conduction electrons form the spin-vortices. The appearance of the non-trivial $\mathbf{A}^{\text {fic }}$ makes the effective vector potential in the system the sum $\mathbf{A}^{\mathrm{eff}}=\mathbf{A}^{\mathrm{fic}}+\mathbf{A}^{\mathrm{em}}$. The effective vector potential $\mathbf{A}^{\mathrm{eff}}$ is gauge invariant and the persistent current is generated by it. The present theory suggests that the reason for the very high superconducting transition temperature in the cuprate is due to an enhanced stability of spin-vortices by the strong hole-lattice interaction.

\section{References}

[1] Anderson P W 1987 Science 2351196

[2] Moriya T and Ueda K 2003 Rep. Prog. Phys. 661299

[3] Lee P A, Nagaosa N and Wen X G 2006 Rev. Mod. Phys. 7817

[4] Maier T A, Jarrell M S and Scalapino D J 2006 Phys. Rev. B 74094513

[5] Maier T A, Jarrell M S and Scalapino D J 2006 Phys. Rev. Lett. 96047005

[6] Ogata M and Fukuyama H 2008 Rep. Prog. Phys. 71036501

[7] Shraiman B I and Siggia E D 1988 Phys. Rev. Lett. 61467

[8] Shraiman B I and Siggia E D 1989 Phys. Rev. Lett. 621564

[9] Vergés J A, Louis E, Lomdahl P S, Guinea F and Bishop A R 1991 Phys Rev. B 436099

[10] Seibold G 1998 Phys Rev. B 5815520

[11] Berciu M and John S 1999 Phys Rev. B 5915143

[12] Timm C and Bennemann K H 2000 Phys. Rev. Lett. 844994

[13] Berciu M and John S 2004 Phys. Rev. B 69224515

[14] Fine B V 2007 Phys Rev. B 75060504.

[15] Koizumi H 2011 J. Supercond. Nov. Magn. 241997

[16] Hidekata R and Koizumi H 2011 J. Supercond. Nov. Magn. 242253

[17] Ando Y, Komiya S, Segawa K, Ono S and Kurita Y 2004 Phys. Rev. Lett. 93267001

[18] Müller K A 2007 J. Phys.: Condens. Matter 19251002

[19] Kresin V Z and Wolf S A 2009 Rev. Mod. Phys. 81481 
[20] Zhang C J and Oyanagi H 2009 Phys Rev. B 79064521

[21] Miyaki S, Makoshi K and Koizumi H 2008 J. Phys. Soc. Jpn. 77034702

[22] LeBoeuf D, Doiron-Leyraud N, Levallois J, Daou R, Bonnemaison J B, Hussey N E, Balicas L, Ramshaw R J, Liang R, Bonn D A, Hardy W N, Adachi S, Proust C and Taillerfer L 2007 Nature 450533

[23] Lanzara A, Bogdanov P V, Zhou X J, Kellar S A, Feng D L, Lu E D, Yoshida T, Eisaki H, Fujimori A, Kishio K, Shimoyama J I, Noda T, Uchida S, Hussain Z and Shen Z X 2001 Nature 412510

[24] Schrödinger E 1926 Ann. Physik 79361

[25] Longuet-Higgins H C, Öpik U, Pryce M H L and Sack R A 1958 Proc. Roy. Soc. London Ser. A 2441

[26] Herzberg G and Longuet-Higgins H C 1963 Disc. Farad. Soc. 3577

[27] Mead C A and Truhlar D G 1979 J. Chem. Phys. 702284

[28] Aharonov Y and Bohm D 1959 Phys. Rev. 115167

[29] Vaknin D, Sinha S K, Moncton D E, Johnston D C, Newsam J M, Safinya C R and King H E 1987 Phys. Rev. Lett. 582802

[30] Berezinskii V L 1972 Sov. Phys. JETP 34610

[31] Kosterlitz J M and Thouless D J 1973 Journal of Physics C: Solid State Physics 61181

[32] Doiron-Leyraud N, Proust C, LeBoeuf D, Levallois J, Bonnemaison J B, Liang R, Bonn D A, Hardy W N and Taillerfer L 2007 Nature 447565

[33] London F 1950 Superfluids vol 1 (New York: Wiley)

[34] Landau L D 1941 J. Phys. U.S.S.R. 571

[35] Anderson P W 1959 Phys. Rev. 1152 